\title{
Facial dysmorphism-developmental delay- behavioral abnormalities syndrome due to 10p11.21p12.31 microdeletion
}

INSERM

\section{Source}

INSERM. (1999). Orphanet: an online rare disease and orphan drug data base. Facial dysmorphism-developmental delay-behavioral abnormalities syndrome due to 10p11.21p12.31 microdeletion. ORPHA:284169

Facial dysmorphism-developmental delay-behavioral abnormalities syndrome due to 10p11.21p12.31 microdeletion is a rare, genetic syndromic intellectual disability characterized by developmental delay, hypotonia, speech delay, mild to moderate intellectual disability, abnormal behavior (autistic, agg ressive, hyperactive) and dysmorphic facial features, including synophrys or thick eyebrows, deep set eyes, bulbous nasal tip and full cheeks. Congenital heart and brain anomalies, visual and hearing impairment are also common. 